\section{Netzwerk Kindersimulation: für den Notfall gewappnet}

Das Netzwerk Kindersimulation wurde 2014 ins Leben gerufen, um alle Personen und Zentren im deutschsprachigen Raum zu verbinden, die sich mit Simulationen in der Pädiatrie beschäftigen.

D ie Simulation von Notfallsituationen hält zunehmend Einzug in die Aus- und Weiterbildung. Dabei werden neben den technischen Fertigkeiten und medizinischen Algorithmen Teamwork, interdisziplinäre Zusammenarbeit und Kommunikation trainiert. Doch bis jetzt waren es meist nur einzelne Personen, die sich für dieses Konzept engagiert haben.

Von nun an sollen durch lokale Vernetzung vorhandene personelle und materielle Ressourcen besser zugänglich gemacht werden. Dem Netzwerk Kindersimulation haben sich bereits 36 Standorte von Lübeck bis nach Südtirol angeschlossen. Die großflächige Vernetzung im gesamten deutschsprachigen Raum erleichtert gemeinsame Forschungsprojekte und ermöglicht die Entwicklung von Standards für die simulationsbasierte Aus- und Weiterbildung.

Die Arbeitsgruppe befasst sich mit Empfehlungen zur Durchführung von simulationsbasierten Lehrmethoden. Ein weiteres Ziel des Netzwerkes ist es außerdem, Qualitätskriterien sowie zertifizierte Aus- und Weiterbildungskurse $\mathrm{zu}$ definieren und umzusetzen. Und schließlich soll ein Mentoren-Pro-

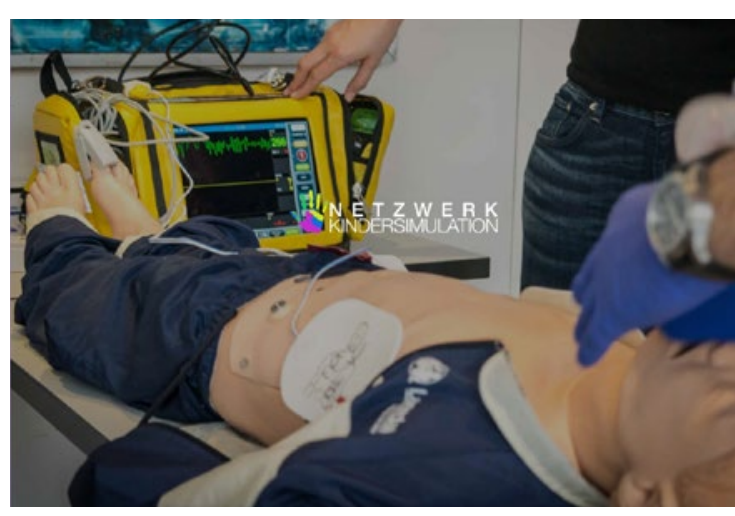

Die Adresse für jeden, der sich mit der Simulation von Notfallsituationen bei Kindern beschäftigt: www.netzwerkkindersimulation.org

gramm sowohl Anfänger als auch Fortgeschrittene im Bereich Kindersimulation unterstützen, um wichtige Erfahrungen auszutauschen.

www.netzwerk-kindersimulation.org

\section{Nicht nur Zuweiser bringen Patienten in die Praxis}

Stimmt der Praxisauftritt im Internet, werden Suchmaschine und damit auch Patienten schnell fündig. Ärzte können dafür leicht in Vorleistung gehen.

$\mathrm{O}$ b nach der Existenzgründung oder für die Expansion der Praxis - wer neue Patienten gewinnen will, kommt um Online-Aktivitäten beim Praxismarketing kaum noch herum. Darauf hat Praxisberaterin Nadja Alin Jung, $\mathrm{m}^{2} \mathrm{c}$ medical concepts \& consulting, beim Seminar „Existenzgründung 2.0" in Berlin hingewiesen. Immer mehr Patienten, die nach einem neuen Arzt suchen, konsultieren dafür das Internet. Dabei spielten Arztbewertungsportale ebenso eine Rolle wie eine Platzierung unter den ersten Einträgen bei der Suchmaschine Google für Leistungen der Praxis, sagte Jung bei der Veranstaltung, die von der Deutschen Apotheker- und Ärztebank gemeinsam mit der Steuerberatungsgesellschaft ETL Advision und Springer Medizin ausgerichtet wurde. „Bei Google auf Seite 1 - da spielt die Musik", betonte die Praxisberaterin. „Daher ist es wichtig, dass Ärzte lernen, mit diesen Medien zu arbeiten".

Die zunehmende Bedeutung von Online-Medien für die Arztsuche zeigt sich auch in Umfragen: 2014 gaben $42 \%$ der Teilnehmer an einer Umfrage unter 1.000 Patienten im Auftrag des Bewertungsportals jameda an, Internet-Suchmaschinen für die Arztsuche zu nutzen, fast jeder dritte recherchierte zu diesem Zweck auf einem Arztbewertungsportal. Nur Freunde und Familie (56\%) und Hausärzte (41\%) waren für die Arztsuche wichtiger oder ebenso wichtig wie Suchmaschinen und Bewertungsportale.

Um bei Google weit vorne gelistet zu werden, sollten Ärzte auf ihrer Website mit Schlüsselworten arbeiten, die zu ihren Spezialleistungen passen, für die sie Patienten gewinnen wollen. Auch substanzielle und verständlich geschriebene aktuelle Informationen zu diesen Leis- tungen helfen, bei Google nach vorne zu kommen. Interessierte Ärzte verfassen die Texte selber, es können aber auch Dienstleister konsultiert werden. Ob die Maßnahmen zum Erfolg führen, kann selbst getestet werden, etwa indem Suchbegriffe wie "Krebsvorsorge Frankfurt" oder „Vasektomie Köln“ oder ähnliches selbst eingegeben werden - je nach Fachgruppe und speziell angebotenen Leistungen. Die Nutzung der eigenen Website lässt sich über Google Analytics recherchieren. Über Google Trends lässt sich erfahren, nach welchen Suchbegriffen wie oft gesucht wird.

Anzeigen bei Google seien dagegen weniger sinnvoll, habe sie festgestellt, sagte Marketing-Expertin Jung in Berlin. Dagegen könne es sehr wohl helfen, zufriedene Patienten anzuregen, die Praxis im Netz auf einschlägigen Portalen zu bewerten. Wichtig sei es auch, regelmäßig zu screenen, wie die Praxis bewertet werde, um gegebenenfalls Missstände abzustellen, auf die Patienten im Internet hinweisen. Hauke Gerlof

Das nächste Seminar Existenzgründung 2.0 für Ärzte findet am 24. Oktober 2015 in München statt: www.apoBank.de/existenzgruendung-2-0 